\title{
ALLEVIATION OF ANTIMICROBIAL THERAPY IN ICU DURING COVID-19 SECOND WAVE - A REVIEW PAPER
}

\author{
Mohamed Nassef \\ Critical Care Medicine \\ AlQassimi Hospital (AQH) - Sharjah, UAE
}

Ahmed A. Shorrab

Anesthesia and Intensive Care, University Hospital Sharjah, UAE

Mohamed Buheji

International Inspiration Economy Project- Bahrain

Abdul Rahman Buheji

Senior House Officer@ Accident \& Emergency

King Hamad University Hospital - Bahrain

Mohammed F. Abosamak

Department of Anesthesia \& ICU, Faculty of Medicine,

Tanta University- Egypt.

\begin{abstract}
The purpose of this paper is to help distinguish between severe COVID-19 and bacterial secondary infections and thus help improve the decision of antibiotic administration during the ICU stay. This study targets to mitigate the risks of the overuse of antibiotics thus avoid further aggravating the antimicrobial resistance.

The authors explored the apparent changes in the antibiogram differentiation due to the way many COVID-19 cases had to be treated with broad-spectrum antibiotics, due to community-acquired pneumonia (CAP), until possible bacterial superinfections had been ruled out. Waves of antibiotic resistance and numbers of extensively drugresistant (XDR) and multidrug-resistant (MDR) bacterial strains and the fatality of patients secondary to sepsis were highlighted in this review.

The implication of the paper is that it calls for more innovative methods that would help to revise and control the antimicrobial stewardship programs in hospitals ICU's. Moreover, it could help to reduce the burden of antibiotic cost without conceding the clinical outcome during the current evolving pandemic and future emergencies.
\end{abstract}


Key words: COVID-19 Patients, Antibiotic Therapy, Community-Acquired Pneumonia (CAP), Hospital-Acquired Pneumonia (HAP), Ventilator-Acquired Pneumonia (VAP), ICU Patients, , Antimicrobial Stewardship Programs

Cite this Article: Mohamed Nassef, Ahmed A. Shorrab, Mohamed Buheji, Abdul Rahman Buheji and Mohammed F. Abosamak, Alleviation of Antimicrobial Therapy in ICU during COVID-19 second wave - A Review Paper, International Journal of Management, 11(12), 2020, pp 2163-2175.

http://iaeme.com/Home/issue/IJM?Volume=11\&Issue=12

\section{INTRODUCTION}

During the past year, the world was exposed to a rapidly growing Pandemic with a new subtle virus; coronavirus 2 (SARS-CoV-2). The infected patients showed a wide spectrum of clinical manifestations ranging from asymptomatic to fatal disease, [1]. The pathogenesis underpinning the infectious process has also included a multitude of factors including direct viral cytopathic effects, immune-based effects with a surge of inflammatory markers and cytokines, coagulopathy and secondary infections. Secondary infections with bacterial or fungal co-infections were reported to be 8 per cent (62 of 806); these included mainly respiratory infections and bacteraemia, [2].

The ambiguity of the disease over the past year, including ethical and resources issues, has produced a situation where antibiotic use was not clear, and the breach of the antibiotic stewardship was imminent. This article was designed to discuss the situation of antibiotic usage in relation to current practices and our regional experience in the management of COVID-19 disease.

No doubt, hospital admissions increase the risk of health-care-associated infections and the transmission of multidrug-resistant organisms, which in turn lead to increased antimicrobial use, Saleem et al. (2019). A recent study conducted in intensive care units in 88 countries showed that although only 54\% $(8135 / 15165)$ of patients had suspected or proven bacterial infection, 70\% (10 640/15 165) of them had received at least one antibiotic either for prophylaxis or treatment purposes, [3].

There are many published articles about antibiotics in COVID 19. Latest studies show that nearly $70 \%$ of COVID-19 patients infected with the novel (SARS-CoV-2) received antibiotic therapy during the hospital stay. The main reason for this might be due to the difficulty in differentially diagnosing whether the symptoms are due to COVID-19 or due to bacterial Community-Acquired Pneumonia (CAP). Till the time of writing of this paper, it is still very challenging to define if the ICU patient fulfilling criteria for CAP, which is positive for SARS-CoV-2 got a bacterial co-infection upon admission. During hospitalisation, it may be difficult to distinguish between severe COVID-19 and bacterial secondary infections. [4].

WHO [5] recommended provision of empirical antimicrobial therapy to prevent superinfection, which will lead to severe acute respiratory infection (SARS) and multi-organ failure in a patient with severe illness. Literature, however, still has many gaps and queries that are unanswered about antimicrobial therapy during the pandemic, which needs to be identified and stratified for the future of humanity. Therefore, this paper targets to investigate some of these gaps that are related to the scope of the research. For example, did the COVID 19 situation cause a huge violation of the antibiotic stewardship and whether this is doing harm by increasing the chance for antimicrobial resistance, [6], [7].

Additionally, what are the current practices that could be retrieved or learned to improve the outcome and clinical experience? Furthermore, what human and financial cost caused by 
antibiotics administration during the pandemic 1st and 2nd waves. Finally, how to improve the use of antibiotics during emergency situations and future pandemics? [8].

As reported in the United Nations report for urgent action to avert the AMR crisis, Irrespective to COVID19, AMR is contributing to more than seven hundred thousand deaths per year, with an estimated total death of up to 10 million by 2050. [9], [10].

The literature shows that despite the lack of evidence of their benefits on COVID-19 infection, a wide range of antiviral, antimicrobial drugs and corticosteroids have been used in the care of COVID-19 patients. One of the early studies was in Wuhan, in China which revealed that $71 \%$ of patients received antibiotics, $25 \%$ of them were treated with combined therapy (more than one antibacterial agent). Others were treated with antiviral agents, [11]. The persistent use of antimicrobials, as shown by the latest research, also showed that it has led to the production of MDR strains and the emergence of AMR. [12], [9].

\section{IMPLICATIONS OF THIS REVIEW}

The aim of this review is to help mitigate the risks of the empirical use of antibiotics during the pandemic and to contain the development of antimicrobial resistance, and thus to limit the direct and hidden costs of antibiotic use. This also should reflect on improving future guidelines in dealing with CAP patients. The review could help improve the antibacterial treatment of hospitalised adults' patients who would have both a respiratory infection and suspected or proven COVID-19 symptoms.

The review takes into consideration how to balance between the challenge of treating lifethreatening diseases such as sepsis, or hospital-acquired infections, and overcoming the threats caused by COVID-19.

\section{LITERATURE REVIEW}

\subsection{COVID-19 and Co-Infections}

\subsubsection{Influence of SARS-CoV-2 on Quality of Treatment}

SARS-CoV-2 can lead to a wide spectrum of disease, ranging from very mild symptoms of upper respiratory tract infection to life-threatening pneumonia. Severe disease is frequently associated with high inflammation markers levels. Many studies confirm that the majority of hospitalised patients with COVID-19 had to be treated with broad-spectrum antibiotics with unknown efficacy. As COVID-19 patients frequently need prolonged hospitalisation and respiratory support, unnecessary antibiotics upon hospitalisation may increase the individual risk of subsequent hospital-acquired pneumonia (HAP) caused by resistant bacteria such as legionella which is the source of infection for Legionnaires' Disease (LD).

Many key human pathogens are showing resistance to antibiotics including Methicillinresistant S. aureus (MRSA), multidrug-resistant Streptococcus, Vancomycin-resistant Enterococci (VRE), resistant Mycobacterium, Carbapenem-resistant Enterobacteriaceae (CRE), Colistin-resistant Klebsiella, Carbapenem-resistant Pseudomonas aeruginosa, and Carbapenem-resistant Acinetobacter baumannii. [7]

With the continuous rise of the hospitalised COVID-19 cases, even with the second wave of the pandemic might lead to a steep increase in antibiotic use and prescriptions for a large number of patients' population. This universal use of antibiotic might trigger an increase in antimicrobial resistance rates to a level never been experienced before. [9].

\subsubsection{When Co-infections Defined?}

COVID-19 patients with secondary co-infections usually diagnosed with (suspected) bacterial pneumonia usually within 48 to 72 hours of admission. 
A severe complication of COVID-19 is viral pneumonia. From experience, distinguishing viral pneumonia from bacterial pneumonia is difficult. However, there are important cues in history and the examination that can help differentiate the two. The clinician should be aware that the coexistence of viral and bacterial pneumonia increases the risk of death. In some cases, they could co-exist, increasing the chance of a more unfortunate outcome. However, there may be important clues in the history and the examination that can help differentiate the two.

Figure (1) shows the High-risk vs low-risk groups in suspected COVID -19 cases, following the work of NICE (2020).

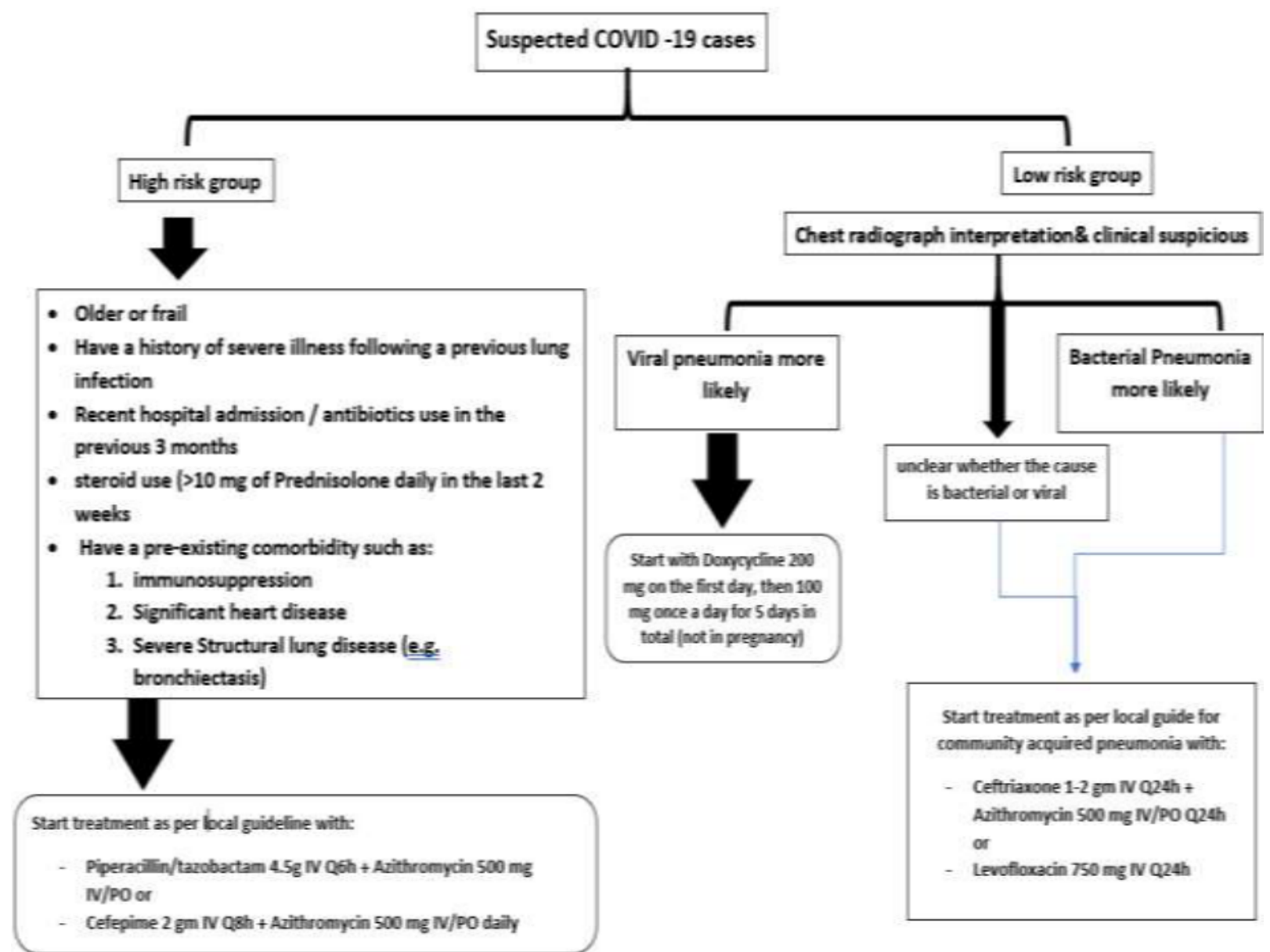

Figure 1

Bacterial community-acquired pneumonia and viral pneumonia may co-exist. A systematic review including 31 studies $(n=10,762$ patients) found that $25 \%$ of patients with CAP had viral infections (95\% CI $22-28 \%$ ), this increased to $44 \%$ in studies where $>50 \%$ had a lower respiratory sample. The review reported that the interaction of CAP and viral infection doubled mortality: odds of death in patients with bacterial and viral infection (OR $2.1,95 \%$ CI 1.32 to 3.31 ) (10 studies). [10]

Therefore, restrictive use of antibacterial drugs in patients who are mild to moderately ill with proven or a high likelihood of COVID-19 could be planned within this time. Exceptions for the restrictive use of antibacterial drugs can be made for patients who present with radiological findings and/or inflammatory markers compatible with bacterial co-infection. Other research team focused on collecting culture of sputum and blood as well as pneumococcal urinary antigen testing before starting of empirical antibiotic therapy. Patients 
with proven or high likelihood of COVID-19 show no bacterial pathogens after 48 hours of incubation. [4]

2019 American Thoracic Society (ATS) / America's Infectious Disease Society (IDSA) guidelines recommended to send sputum culture and blood culture in patients with severe CAP as well as in all inpatients empirically treated for MRSA or Pseudomonas aeruginosa. Additionally, emphasis on local epidemiology and validated risk factors to determine the need for MRSA or P. aeruginosa coverage and increased emphasis on de-escalation of treatment if cultures are negative. They also recommend that empiric antibiotic therapy should be initiated in adults with clinically suspected and radiographically confirmed CAP regardless of initial serum procalcitonin level, [14].

The study of Metlay et al. [14] (2019) lead to the development of a predictive bedside model and scoring system in 103 consecutive patients classified as having virus-like (48), bacterial (37) and unknown (18) pneumonia found that the independent predictors for bacterial pneumonia were: acute onset of symptoms (OR 31; 95\% CI, 6-150), age $>65$, comorbidity (OR 6.9; 95\% CI, 2-23), Leukocytosis or leukopenia (OR 2; 95\% CI, 0.6-7). The sensitivity and specificity of the score to identify patients with bacterial pneumonia were $89 \%$ and $94 \%$, respectively. The sample was small, and the results might not be generalisable outside of the ED setting, and they have not been validated.

A meta-analysis included 12 studies of 2408 adult patients with documented bacterial versus non-bacterial aetiology of CAP were conducted by Kamat et al. [15]. For the 8 studies using a procalcitonin cut-off of $0.5 \mu \mathrm{g} / \mathrm{L}$, the pooled sensitivity and specificity estimates were $55 \%$ (95\% CI, 37-71\%) and 76\% (95\% CI, 62-86\%), respectively. The review found that the sensitivity and specificity were both too low and variable for the results to be confidently used in the decision-making. Figure (2) shows a summary of how CAP can be dealt with. Kamat et al. [15]

Figure (2) Illustrate the type of CAP cases Antibiotics protocols in both ICU and NonICU patients

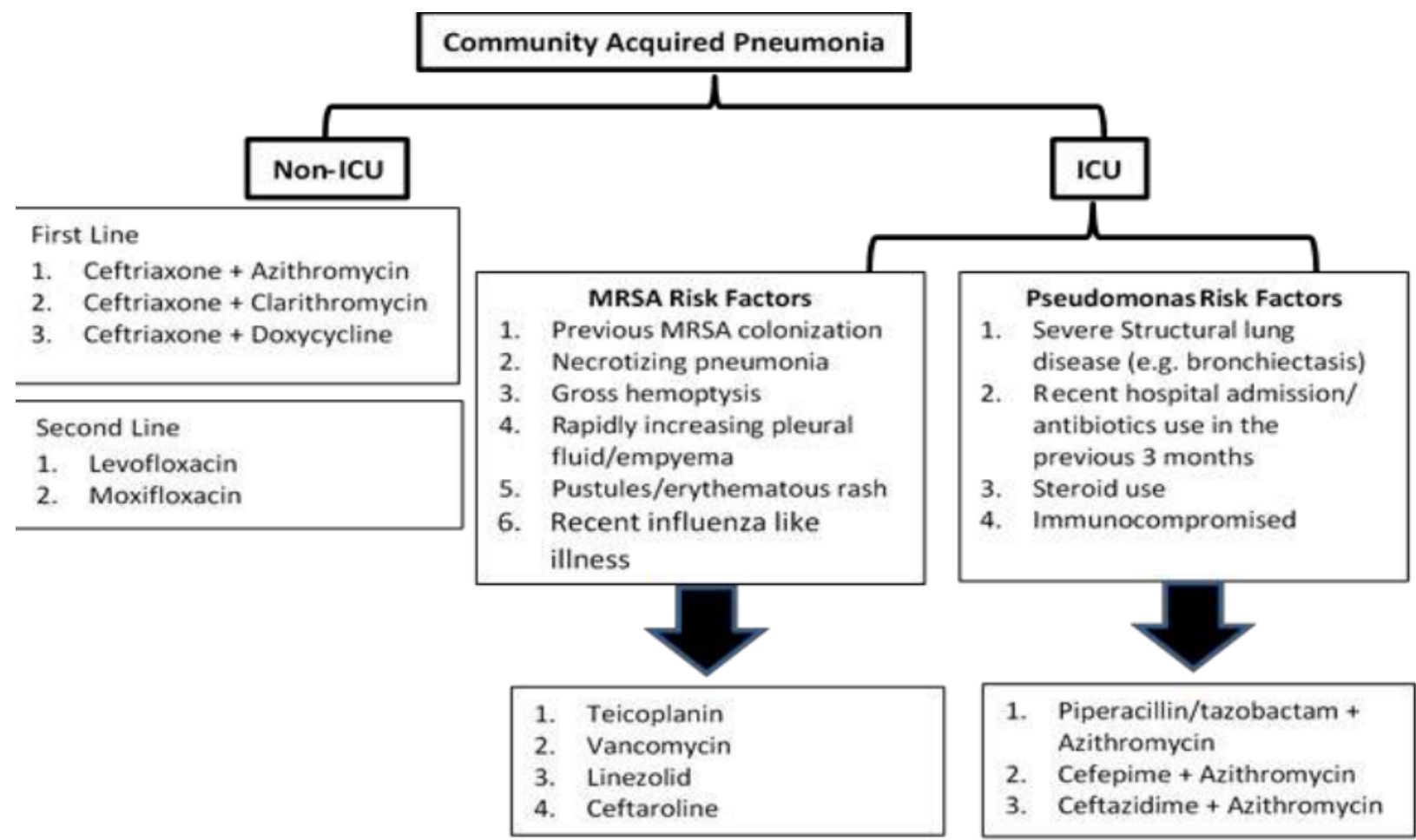

Figure 2 


\subsubsection{Bacterial Pneumonia or Bacteraemia in Ventilated Patients}

There are studies that were carried out in different countries, including Wuhan in China shows that secondary infections found in 10\% of the hospitalised COVID-19 patients. Most of the incidences of nosocomial, microbiologically-confirmed bacterial pneumonia or bacteraemia. [16], [19]

Langford and his team [17] did a systematic review and meta-analysis on bacterial infections in COVID-19 patients. $7.1 \%$ of the 28 eligible COVID-19 patient cases studies found to have bacterial infections. $3.5 \%$ of the cases in the studies found to have bacterial coinfections.

Other studies show that secondary infections (HAP; or bacteraemia) were associated with pneumonia (VAP) that came from the ventilator. Rawson et al. [2] showed as a result of the systematic review that $8 \%$ of the COVID-19 patients have bacterial and fungal co-infections at any time during hospitalisation or ventilation. Figure (3) shows the differential diagnosis protocol for HAP/VAP cases. [19], [20].

Figure (3) Illustrates the Proposed Anti-biotics Protocols for Hospital-Acquired Pneumonia (HAP) and Ventilator-Acquired Pneumonia (HAP) Patients

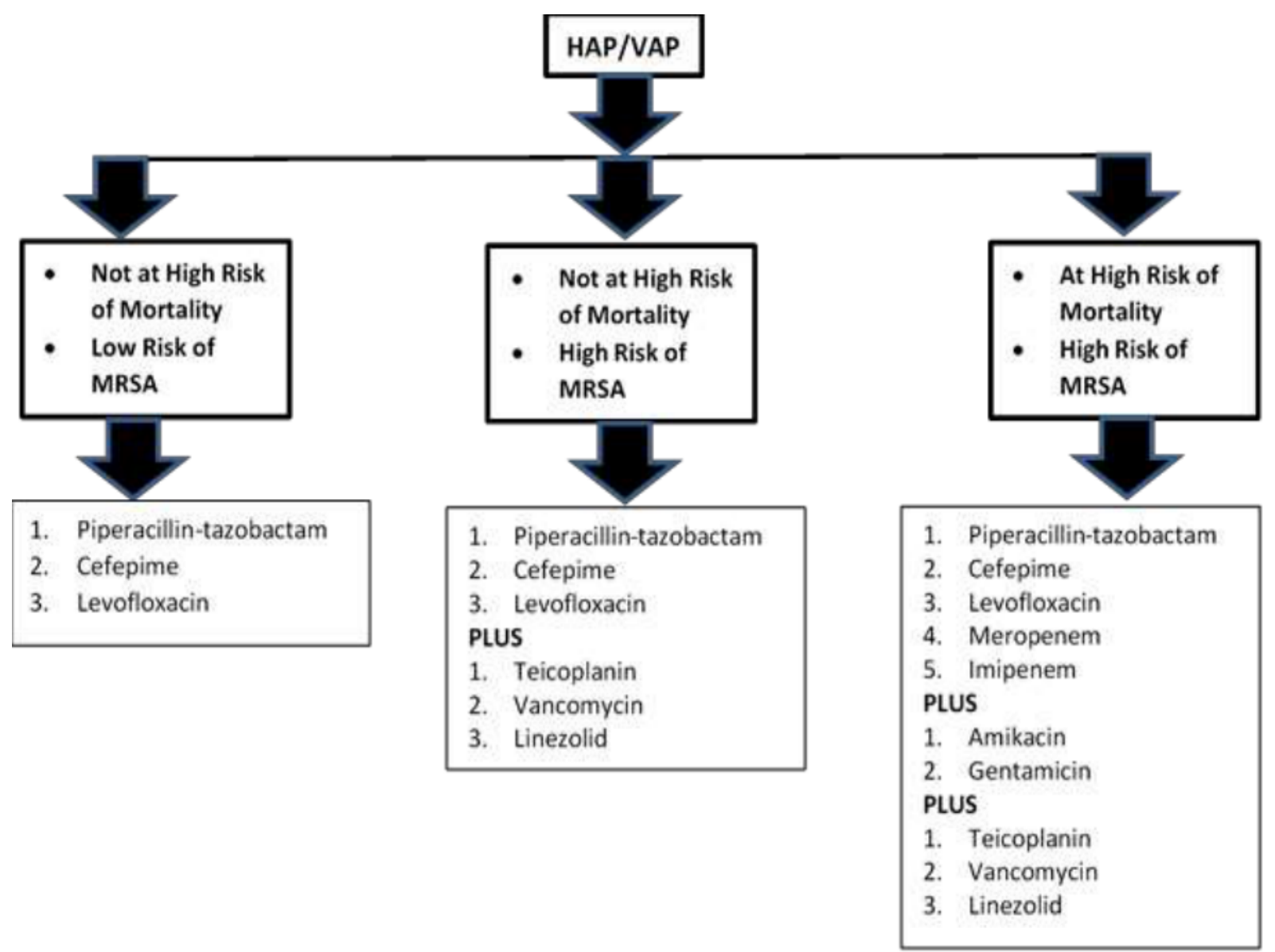

Figure 3

\subsubsection{Type of Bacterial Species in COVID-19 \& Bacterial Pneumonia Patients with Proven or High Likelihood of COVID-19}

The pathogens reported in COVID-19 patients with possible bacterial co-infection were mainly Staphylococcus aureus, Haemophilus influenzae, Streptococcus pneumoniae, 
Klebsiella pneumoniae and Acinetobacter baumannii were isolated from respiratory specimens, [11].

The systematic review and meta-analysis of Langford et al. [17] reported identified bacteria in 188 studies on bacterial infections in COVID-19 patients.

\subsection{Differential Diagnosis}

\subsubsection{Differential Diagnosis between COVID-19 and Bacterial Community-Acquired Pneumonia}

Since the outbreak of COVID-19 pandemic, this new disease is still causing confusion to many clinicians whether if the symptoms are related to the COVID-19 or to bacterial community-acquired pneumonia. COVID-19 patients often develop a hyperinflammatory phase during the disease process, which could again be difficult to distinguish from a secondary bacterial infection. Community-acquired pneumonia (CAP) has caused more repel effect of a prolonged increase in hospitalisations of patients. [18]

Besides the flowchart of Figure (1), Table (1) could help to do more differentiation following the symptoms or signs of the patients and whether they could be developed either COVID pneumonia or a Bacterial Pneumonia.

Table 1 Further Differential Diagnosis Input between COVID pneumonia and Bacterial Pneumonia

\begin{tabular}{|c|c|c|}
\hline & COVID pneumonia more likely if & Bacterial Pneumonia more likely if \\
\hline $\begin{array}{l}\square \\
\square \\
\square \\
\square \\
\square\end{array}$ & $\begin{array}{l}\text { Presents with a history of typical } \\
\text { COVID-19 symptoms for about a week } \\
\text { has severe muscle pain (myalgia) } \\
\text { has a loss of sense of smell (anosmia) } \\
\text { is breathless but has no pleuritic pain } \\
\text { has a history of exposure to known or } \\
\text { suspected COVID-19, such as a household } \\
\text { or workplace contact? }\end{array}$ & $\begin{array}{l}\square \quad \text { becomes rapidly unwell after only a few } \\
\text { days of symptoms } \\
\square \quad \text { does not have a history of typical } \\
\text { COVID-19 symptoms } \\
\square \quad \text { has pleuritic pain } \\
\square \quad \text { has purulent sputum }\end{array}$ \\
\hline $\begin{array}{l}\square \\
\square \\
\square \\
\square \\
\square \\
\square\end{array}$ & $\begin{array}{l}\text { Insidious onset } \\
\text { Lower temperature } \\
\text { Tachycardia or tachypnoea out of } \\
\text { proportion to the temperature } \\
\text { A paucity of physical findings on } \\
\text { pulmonary exam disproportionate to the } \\
\text { level of disability } \\
\text { Bilateral positive lung finding }\end{array}$ & $\begin{array}{ll} & \text { Acute onset } \\
\square & \text { Higher temperature } \\
\square & \text { Unilateral positive lung findings }\end{array}$ \\
\hline
\end{tabular}

\subsubsection{Diagnosing through Procalcitonin}

Procalcitonin (PCT) has been shown to be useful in the early diagnosis of lower respiratory tract infections of bacterial origin. Also, measuring PCT levels can help determine the need for antibiotic therapy. C-reactive protein (CRP) is usually increased in COVID-19 patients, while PCT is usually low. But in patients with severe COVID-19 disease, the diagnosis becomes more difficult as the secondary bacterial infections increase and thus, the PCT levels found to increase. [4].

According to Sieswerda et al. [4]; 50\% of the ICU patients developed a secondary infection during ICU admission, and those shown a significant increase in both PCT and CRP. 
COVID-19 patients who do not develop bacterial infections and have high initial CRP levels and low to moderate PCT levels, found to have CRP decrease gradually decrease over time. [4].

\subsubsection{Importance of Biomarkers to Predict Secondary Infection}

In order to help improve the differential diagnosis between the COVID-19 and/or prediction of the secondary infections in ICU patients, biomarkers were suggested. By monitoring PCT levels, initiation of antibacterial therapy in ICU patients can be controlled. CRP in COVID-19 patients is consistently elevated; therefore, it does not have the predictive value for bacterial infections in the initial phase. However, with biomarkers monitoring both PCT and CRP may help identify or rule out secondary bacterial infections and facilitate the appropriate use of antibiotic therapy, where necessary. [4], [13].

\section{DISCUSSION}

\subsection{Role of Differential Diagnosis in refuting Bacterial Pneumonia in patients with a proven or high likelihood of COVID-19}

The reviews by Wynants et al. [13] who did meta-analysis summarised 18 out of 31 studies focused on the importance of five types of general prediction models for the differential diagnosis of COVID-19 patients. The Wynants team seen that the most common predictors were clinical or demographical factors, such as age, fever and other signs and symptoms. The rest of the predictions models were dependent on the CT-scan assessment.

A more developed differential diagnosis is needed to distinguish the viral from bacterial pneumonia, which could help to mitigate the risk of a bacterial co-infection in patients with suspected COVID-19; this was also supported with recent America's Infectious Disease Society (IDSA) guideline where procalcitonin should not be used in the decision to start or withhold antibiotics in patients with CAPM, [14].

In a nutshell, the combination of the clinical course of disease and results obtained from laboratory tests and imaging are leading in the assessment of the likelihood of bacterial coinfection in patients with COVID-19.

\subsection{When to Consider Antibiotic Therapy}

Antibiotic therapy could be considered if bacterial co-infection is found in a patient with radiological findings and/or inflammatory markers compatible with bacterial co-infection. Patients with a proven or high likelihood of COVID-19 can start empirical antibiotic therapy while awaiting diagnostic test results, if they were severely immunocompromised, i.e. on chemotherapy, or having poorly controlled HIV.

In general, there are mainly four of patients that are considered of the more at risk bacterial co-infection include:

1 -The very young and the elderly (there is a decline in incidence from adolescence through to the fifth decade).

2-Age-related immunosuppression

3-Age-related comorbidities.

4-Immunosuppressive therapy and secondary impaired immunities

Disease-modifying haematological/immunological agents in chronic illness. 


\subsection{Strategies Managing Co-infections in Severely III Patients}

Up to $20 \%$ of secondary infections in COVID-19 patients, are found in severely ill patients. Studies show a gap in antibacterial strategies effective outcomes of secondary infections in COVID-19 patients.

One of the strategies used is the selective decontamination of the digestive tract, after obtaining cultures, in COVID-19 patients with suspected secondary bacterial respiratory infection with HAP and/or VAP. Another strategy is the use of procalcitonin before the initiation of antibiotic therapy for COVID-19 patients. However, this strategy does not have enough scientific evidence yet. [4].

Tabah et al. [6] called for setting an appropriate de-escalation strategy to reduce unnecessary antibiotic use as much as possible in COVID-19 patients. For example, the Tabah team suggested to stop the antibiotics, even if they have been started, in patients that no pathogens in representative sputum, blood culture and urinary antigen tests, after 48 hours of incubation.

Antibiotic treatment duration of five days is likely to be sufficient in patients with COVID-19 and suspected bacterial co-infection upon the improvement of signs, symptoms and inflammatory markers. Therefore, Metlay et al. [14] see that that procalcitonin levels could be used to support shortening the duration of antibacterial therapy.

Bacterial pathogens in patients with a co-infection seemed similar to those report with regular bacterial CAP, [6]. Therefore, it is advisable to monitor the guidelines on CAP and try to modify it according to the preferred severity of the disease.

2019 ATS/IDSA Guidelines recommended sputum culture and blood culture in patients with severe Community-acquired Pneumonia as well as in all inpatients empirically treated for MRSA or Pseudomonas aeruginosa. Additionally, emphasis on local epidemiology and validated risk factors to determine the need for MRSA or P. aeruginosa coverage and increased emphasis on de-escalation of treatment if cultures are negative.

They also recommend that empiric antibiotic therapy should be initiated in adults with clinically suspected and radiographically confirmed CAP regardless of initial serum procalcitonin level. [6]

\subsection{Comparing the Reviews with the Facts from the Second Wave of COVID-19}

Based on the current, the vast majority of COVID-19 patients with a respiratory illness does not always develop bacterial co-infection. However, based on the currently available evidence and antibiotic stewardship development during the pandemic, more restrictive use of antibacterial drugs in patients with a community-acquired respiratory infection and proven or high likelihood of COVID-19 need to be applied in the future. [21], [22].

Nosocomial hospital-acquired infections are commonly observed in ICU patients infected with a SARS-CoV-2 virus. However, the prophylactic use of antibiotics can contribute to the AMR crisis and makes antibiotics ineffective when patients acquire antibiotic-resistant strains, [23].

The learning for a future pandemic is that more clinical assessments need to be taken on patients with mild or moderately-severe respiratory disease, before any antibiotic's usage. The usage should be balanced with the probability that patients could acquire antibiotics resistance and get secondary infections by MDR organisms. This should be part of the differential diagnosis protocols. 


\section{CONCLUSION AND RECOMMENDATION}

This review emphasises on a learning point for a future pandemic, which is the importance of clinical assessments in patients with mild or moderately severe respiratory disease, before any antibiotics administration. The antibiotics administration should be balanced with the probability that pathogens could become antibiotics resistant, and the chances of getting secondary infections by MDR organisms increase.

Many of the reported studies required more restrictions on antibacterial treatment when dealing with bacterial co-infections other than respiratory pneumonia infection in the patient of a proven or high likelihood of COVID-19. One of the main lessons from the pandemic is the gap for more dynamic yet effective protocols that would minimise the overuse of antibiotics for the overlapping infection symptoms in hospitalised patients.

More research needs to be taken to explore how to improve the current differential diagnosis outcome and thus, the overall clinical effectiveness, which might also reduce also the direct and hidden cost. This would lead to the reduction of lost opportunities and the economic return of antibiotics utilisation, especially during future pandemics.

Besides the regular update of recommendations on treatment in COVID-19 patients, there are really no clear guidance on steps for differential diagnosis for bacterial co-infections, secondary infections that would help in the optimal management of COVID-19 patients. Studies supporting the restrictive antibiotic use from antibiotic stewardship need to give alternative solutions for bacterial infections in COVID-19 patients. [21], [22].

Unfortunately, microbiological testing has yet to deliver fast, accurate, and affordable testing that results in proven benefit for patients with CAP in terms of more rapid delivery of targeted therapy or safe de-escalation of unnecessary therapy. Exceptions include rapid testing for MRSA and influenza. Until we have such widely available (and affordable) tests, therapy for many or most patients with CAP will remain empiric. [6]

This paper confirms what Zumla et al. [24] confirmed, where patients with respiratory tract infection at any point of care, would need rapid diagnostic tests from the respiratory tract that would help distinguish bacterial from a viral infection, and identify any bacteria and delineate antibiotic sensitivities. These tests would enable prompt initiation of pathogenspecific treatment, or enable the prompt modification of empiric antibiotic therapy, and thus improve management and outcomes of patients presenting with respiratory tract infection.

A key to optimal antimicrobials administration is rapid, accurate and affordable tests for pathogens identification. Rapid identification of the infecting pathogen will benefit the patients by guiding the timely administration of the proper antimicrobial drugs. Moreover, rapid testing allows for safe de-escalation of the empirical antimicrobials when the infecting pathogens are identified. Testing for MRSA and influenza are good examples of rapid testing, however, until such rapid tests become widely available and affordable, most patients with CAP will continue to receive empirical antimicrobial treatment, [24].

Minimising unnecessary empirical antimicrobials administration can be achieved through a rapid point of care testing of COVID19 via the integration of the RT-LAMP method with optical and nanomaterial-based system and/or advanced information technologies, [25]. Thus, justifying the administration of specific antiviral treatments. Guidelines should restrict antibiotic treatment for future pandemics to minimise the expected economic burden.

The main limitation of this study is that it did not measure the effectiveness of antibiotics regimens in COVID-19 patients with suspected bacterial pneumonia. The other limitation of this study is that it did not make a distinction between co-infections upon admission and secondary infections that occurred during hospitalisation. This can be compensated in any future differential diagnosis protocol development. 
Finally, the other implication of this paper is that clarifies the need for a contemporary cost-benefit analysis for antibiotics in the era of COVID 19 pandemic. The analysis entails three cost components: cost of the drug and administration; the cost of side-effects; and costs of future resistance. 'No treatment' incurs no costs compounded by evidence and ethical concerns.

\section{REFERENCES}

[1] Wu Z, McGoogan JM. (2020) Characteristics of and Important Lessons from the Coronavirus Disease 2019 (COVID-19) Outbreak in China: Summary of a Report of 72314 Cases from the Chinese Center for Disease Control and Prevention. JAMA. Apr 7;323(13):1239-1242.

[2] Rawson T.M., Moore L.S.P., Zhu N., Ranganathan N., Skolimowska K., Gilchrist M. (2020) Bacterial and fungal co-infection in individuals with coronavirus: a rapid review to support COVID-19 antimicrobial prescribing. Clin Infect Dis: Off Publ Inf Dis Soci Am.

[3] Kampf G. (2018) Biocidal agents used for disinfection can enhance antibiotic resistance in gram-negative species. Antibiotics (Basel). Dec 14;7(4):110.

[4] Sieswerda, E; de Boer, M; Bonten, M; Boersma, W; Jonkers, R; Aleva, R; Kullberg, B; Schouten, J; de Garde, E; Verheij, T; der Eeden, M; Prins, J and Wiersigna, W (2020) Recommendations for antibacterial therapy in adults with COVID-19 - an evidence based guideline. Public Health Emergency Collection, Clinical Microbiology Infection, Oct 1, S1198-743X(20)30594-2.

[5] WHO (2020a) Clinical management of severe acute respiratory infection when COVID-19 is suspected. interim guidance, 28 January 2020. World Health Organization. https://apps.who.int/iris/handle/10665/330893 Accessed: 1/11/2020

[6] Tabah A., Bassetti M., Kollef M.H., Zahar J.R., Paiva J.A., Timsit J.F. (2019) Antimicrobial de-escalation in critically ill patients: a position statement from a task force of the European society of intensive Care medicine (ESICM) and European society of clinical Microbiology and infectious diseases (ESCMID) critically ill patients study group (ESGCIP) Int Care Med.

[7] Kumar, A., and Chordia, N. (2017). "Bacterial resistance against antibiotics," in Drug Resistance in Bacteria, Fungi, Malaria, and Cancer, eds G. Arora, A. Sajid, and V. Kalia (Cham: Springer), 171-192.

[8] Health Management (2020) COVID-19 and Antimicrobial Stewardship. Mon, 19 Oct. https://healthmanagement.org/c/icu/news/covid-19-and-antimicrobial-stewardship Accessed: 9/12/2020

[9] WHO (2019) Interagency coordination group on antimicrobial resistance (IACGs). No time to wait: securing the future from drug-resistant infections. Report to the Secretary-General of the United Nations. https://www.who.int/antimicrobial-resistance/interagency-coordinationgroup/IACG_final_report_EN.pdf?ua=1, Accessed: 1/11/2020

[10] Burk, M; El-Kersh, K; Saad, M; Wiemken, T; Ramirez, J; Cavallazzi, R (2016)Viral infection in community-acquired pneumonia: a systematic review and meta-analysis, European Respiratory Review 2016 25: 178-188;

[11] Chen N., Zhou M., Dong X., Qu J., Gong F., Han Y. (2020) Epidemiological and clinical characteristics of 99 cases of 2019 novel coronavirus pneumonia in Wuhan, China: a descriptive study. Lancet (London, England);395:507-513.

[12] Mulani MS, Kamble EE, Kumkar SN et al. (2019) Emerging strategies to combat ESKAPE pathogens in the era of antimicrobial resistance: a review. Front Microbiol; 10: 539.

[13] Wynants L., Van Calster B., Bonten M.M.J., Collins G.S., Debray T.P.A., De Vos M. (2020) Prediction models for diagnosis and prognosis of covid-19 infection: systematic review and critical appraisal. BMJ (Clinical Research ed.) ;369:m1328. 
[14] Metlay J.P., Waterer G.W., Long A.C., Anzueto A., Brozek J., Crothers K. (2019) Diagnosis and treatment of adults with community-acquired pneumonia. Off Clin Pract Guideline Am Thor Soc Inf Dis Soc Am, Am J Respirat Crit Care Med.;200: e45-e67.

[15] Kamat IS, Ramachandran V, Eswaran H, Guffey D, Musher DM. (2020) Procalcitonin to Distinguish Viral from Bacterial Pneumonia: A Systematic Review and Meta-analysis. Clin Infect Dis. 2020 Jan 16;70(3):538-542. doi: 10.1093/cid/ciz545. PMID: 31241140.

[16] Jean, S. S., Chang, Y. C., Lin, W. C., Lee, W. S., Hsueh, P. R., \& Hsu, C. W. (2020). Epidemiology, Treatment, and Prevention of Nosocomial Bacterial Pneumonia. Journal of clinical medicine, 9(1), 275. https://doi.org/10.3390/jcm9010275

[17] Langford B.J., So M., Raybardhan S., Leung V., Westwood D., MacFadden D.R. (2020) Bacterial co-infection and secondary infection in patients with COVID-19: a living rapid review and meta-analysis. Clin Microbiol Inf: Off Publ Eu Soci Clin Microbiol Inf Dis.

[18] Freeman, A and Leigh, $\mathrm{T}$ (2020) Viral Pneumonia, November 19. https://www.ncbi.nlm.nih.gov/books/NBK513286/Accessed: 9/12/2020

[19] Buheji, M; Cunha, K; Rocha, R (2020) Ventilators In Covid-19, Between Scarcity And Abundance Mindset, International Journal of Advanced Research in Engineering and Technology (IJARET) Volume 11, Issue 10, October 2020, pp. 751-767.

[20] Masterton, R; Galloway, G; French, M; Street, J; Armstrong, E; Brown, J; Cleverley, P; Dilworth, C; Fry, A; Gascoigne, D; Knox, A; Nathwani, D; Spencer, R; Wilcox, R (2008) Guidelines for the management of hospital-acquired pneumonia in the UK: Report of the Working Party on Hospital-Acquired Pneumonia of the British Society for Antimicrobial Chemotherapy, Journal of Antimicrobial Chemotherapy, Volume 62, Issue 1, July, p. 5-34.

[21] Borek AJ, Wanat M, Sallis A et al. (2019) How can national antimicrobial stewardship interventions in primary care be improved? A stakeholder consultation. Antibiotics; 8: 207.

[22] Laxminarayan R, Matsoso P, Pant S et al. (2016) Access to effective antimicrobials: a worldwide challenge. Lancet; 387: 168-75.

[23] Zhou, F., Yu, T., Du, R., Fan, G., Liu, Y., Liu, Z., et al. (2020) Clinical course and risk factors for mortality of adult inpatients with COVID-19 in Wuhan, China: a retrospective cohort study. Lancet 395, 1054-1062.

[24] Zumla, Alimuddin, et al. (2014) Rapid point of care diagnostic tests for viral and bacterial respiratory tract infections - needs, advances, and future prospects. The Lancet Infectious Diseases 14.11 (2014): 1123-1135.

[25] Augustine, R., Hasan, A., Das, S., Ahmed, R., Mori, Y., Notomi, T., . \& S Thakor, A. (2020). Loop-mediated isothermal amplification (Lamp): A rapid, sensitive, specific, and costeffective point-of-care test for coronaviruses in the context of covid-19 pandemic. Biology, 9(8), 182.

[26] Buheji, M (2020) Mitigating Risks of Legionella Outbreak with Re-Opening Post-COVID-19 Pandemic, International Journal of Advanced Research in Engineering and Technology (IJARET) Volume 11, Issue 11, November, pp. 1614-1627.

[27] de Kraker ME, Stewardson AJ, Harbarth S. (2016) Will 10 million people die a year due to antimicrobial resistance by 2050? PLoS Med; 13: e1002184.

[28] Kalil AC, Metersky ML, Klompas M, Muscedere J, Sweeney DA, Palmer LB, Napolitano LM, O'Grady NP, Bartlett JG, Carratalà J, El Solh AA, Ewig S, Fey PD, File TM Jr, Restrepo MI, Roberts JA, Waterer GW, Cruse P, Knight SL, Brozek JL. (2016) Management of Adults with Hospital-acquired and Ventilator-associated Pneumonia: 2016. Clinical Practice Guidelines by the Infectious Diseases Society of America and the American Thoracic Society. Clin Infect Dis. 2016 Sep 1;63(5):e61-e111. 
[29] Huttner B., Catho G., Pano-Pardo J.R., Pulcini C., Schouten J. (2020) COVID-19: don't neglect antimicrobial stewardship principles! Clin Microbiol Inf: Off Publ Eu Soc Clin Microbiol Inf Dis.

[30] NICE (2020) COVID-19 rapid guideline: managing suspected or confirmed pneumonia in adults in the community, National Institute for Health and Care Excellence guideline [NG165], 03 April. https://www.nice.org.uk/guidance/ng165 Accessed on: 1/12/2020

[31] Saleem Z, Godman B, Hassali MA, Hashmi FK, A Rehman, IU (2019) Point prevalence surveys of health-care-associated infections: a systematic review. Pathog Glob Health. 2019 06;113(4):191-205.

[32] Van Berkel M, Kox M, Frenzel T et al. (2020) Biomarkers for Antimocorbial Stewardship: a Re-appraisal in COVID-19 times? Critical Care 24, 600.

[33] WHO (2020b) Clinical management of COVID-19. World Health Organization. interim guidance, 27 May 2020. https://www.who.int/publications/i/item/clinical-management-ofcovid-19, Accessed: $1 / 11 / 2020$ 